\title{
Numerical modeling of the global ionospheric effects of storm sequence on September 9-14, 2005-comparison with IRI model
}

\author{
M. V. Klimenko ${ }^{1,2}$, V. V. Klimenko ${ }^{1}$, K. G. Ratovsky ${ }^{3}$, and L. P. Goncharenko ${ }^{4}$ \\ ${ }^{1}$ N.V. Pushkov IZMIRAN RAS, West Department, Kaliningrad, 236010, Russia \\ ${ }^{2}$ Kaliningrad State Technical University, Kaliningrad, Russia \\ ${ }^{3}$ Institute of Solar-Terrestrial Physics SB RAS, Irkutsk, Russia \\ ${ }^{4}$ Haystack Observatory, Massachusetts Institute of Technology, Westford, Mass., U.S.A.
}

(Received May 31, 2010; Revised April 14, 2011; Accepted June 27, 2011; Online published July 27, 2012)

\begin{abstract}
This study presents the modeling of ionospheric response to geomagnetic storms of September 9-14, 2005. We examine the performance of the Global Self-Consistent Model of Thermosphere, Ionosphere and Protonosphere (GSM TIP) and International Reference Ionosphere-2000 (IRI-2000), and compare the modeling predictions with the ionosonde and incoherent scatter radar observations over Yakutsk, Irkutsk, Millstone Hill and Arecibo stations. IRI-2000 predicted well all negative $f_{\mathrm{o}} F_{2}$ disturbances. In comparison with IRI-2000, the GSM TIP better reproduced the positive phase observed during the disturbed times. We discuss the possible reasons of the differences between the GSM TIP model calculations, IRI predictions, and the observations.
\end{abstract}

Key words: Ionospheric storm, $f_{\mathrm{o}} F_{2}$ disturbances, thermospheric wind, neutral atmosphere composition, electric field, numerical modeling, IRI model.

\section{Introduction}

A large number of studies were devoted to modeling of the ionospheric effects of geomagnetic storms (see, e.g., a review by Buonsanto (1999) and references in Klimenko et al. (2011)). The use of numerical modeling for the description of the ionospheric behavior during geomagnetic disturbances allows investigation of the physical mechanisms responsible for the ionospheric disturbances. In this paper, we discuss the role of each possible mechanism of ionospheric disturbance during geomagnetic storms. A physical mechanism for positive ionospheric storms has recently been suggested by Balan et al. (2009, 2010). Note that Balan et al. (2009, 2010) used empirical models of the thermospheric parameters and the measured electric fields as input parameters for their ionospheric model. Such an approach takes into account the influence of the thermospheric parameters and electric field on the behavior of ionospheric parameters.

However, such study does not consider the inverse effect (i.e., the ionospheric influence on the thermospheric parameters and on the electric field), which in some cases may be very important. The magnetospheric processes act to produce a number of interesting ionospheric features, including electron and ion temperature hot spots, large-scale plasma blobs, extended tongues of ionization, ionization troughs and peaks, and super plasma fountain. These ionospheric features then affect the thermospheric structure, circulation, and composition owing to ion-neutral momentum and energy coupling, and these changes, in turn, affect the

Copyright (C) The Society of Geomagnetism and Earth, Planetary and Space Sciences (SGEPSS); The Seismological Society of Japan; The Volcanological Society of Japan; The Geodetic Society of Japan; The Japanese Society for Planetary Sciences; TERRAPUB.

doi:10.5047/eps.2011.06.048 ionosphere (Schunk, 1990). However, the impact of the ionosphere on the neutral density and wind structures is not well understood. A fully self-consistent thermosphereionosphere-electrodynamics model is required for a comprehensive understanding and modeling capability of the impact of the ionosphere on neutral density and wind structures (Forbes, 2007). The simulation results (Maruyama et al., 2003) suggested that ion drag parallel to the field lines, in the vicinity of a pronounced equatorial ionization anomaly, has a significant impact on the latitudinal structure of the equatorial neutral wind and on the temperature structure. Fully self-consistent thermosphere-ionosphereelectrodynamics models were used recently (e.g., Lu et al., 2008; Klimenko et al., 2011) for understanding of formation mechanisms of ionospheric disturbances during geomagnetic storms.

In parallel to physics-based models investigating the ionospheric effects of geomagnetic storms, empirical models have been developed. The well-known International Reference Ionosphere model (IRI-2000) (Bilitza, 2001, 2003) contains a geomagnetic activity dependence based on an empirical storm-time ionospheric correction model. In the present paper we examine both the Global Self-Consistent Model of the Thermosphere, Ionosphere, Protonosphere (GSM TIP) (Namgaladze et al., 1988, 1991) calculations and the IRI-2000 predictions of the ionospheric effects of the September 9-14, 2005 geomagnetic storm sequence by comparison with experimental data at different latitudes and longitudes. For this purpose we have selected the ionosonde and Incoherent Scatter Radar (ISR) (where available) observations at Yakutsk, Russia $\left(62.0^{\circ} \mathrm{N}\right.$, $129.4^{\circ} \mathrm{E}$; geomagnetic coordinates $51.1^{\circ}, 194.2^{\circ}$ ), Irkutsk, Russia $\left(52.2^{\circ} \mathrm{N}, 104.2^{\circ} \mathrm{E}\right.$; geomagnetic coordinates $40.9^{\circ}$, 
$\left.175.1^{\circ}\right)$, Millstone Hill, U.S.A. $\left(42.6^{\circ} \mathrm{N}, 71.5^{\circ} \mathrm{W}\right.$; geomagnetic coordinates $54.0^{\circ}, 357.9^{\circ}$ ), and Arecibo, Puerto Rico $\left(18.3^{\circ} \mathrm{N}, 66.8^{\circ} \mathrm{W}\right.$; geomagnetic coordinates $\left.29.7^{\circ}, 3.3^{\circ}\right)$.

\section{Basic Physical Mechanisms of Ionospheric Dis- turbances}

It is well known that the primary formation mechanisms of ionospheric disturbances are due to the variations in the electric fields and in the thermospheric parameters. According to the pioneering work by Mayr and Volland (1973), the middle latitude positive ionospheric disturbances are formed by the meridional component of the thermospheric wind, and the negative disturbances are due to the thermosphere composition variations.

It is also known (Rishbeth and Garriott, 1969) that the occurrence of the additional eastward (westward) electric field leads to the additional electromagnetic drift directed to pole and upward (to equator and downward) in the plane of the geomagnetic meridian, and to the plasma transport upward (downward) to the larger (lower) heights into the region of smaller (larger) rates of chemical losses that lead to the positive (negative) effects in the ionospheric $F$-region electron density.

The appearance of the additional meridional electric field leads to the zonal electromagnetic plasma drift. A poleward electric field leads to a westward plasma drift, and an equatorward electric field causes a eastward plasma drift. The zonal $\mathbf{E} \times \mathbf{B}$ drift leads to a change in the electron density in the $F$-region of the ionosphere only in locations with longitudinal gradients in the electron density (Klimenko and Namgaladze, 1980). The magnitude of effects in the electron density will depend on the magnitude of these gradients. The most significant effect of the meridional electric field is expected near the solar terminator.

Since the $F$-region plasma is magnetized it can move across geomagnetic field lines only under the action of an electric field, the effects of which are considered above. Because of the inclination of geomagnetic field to the Earth's surface the meridional component of thermospheric wind can move the plasma due to ion-neutral collisions, transporting it upwards (downwards) along geomagnetic field lines into the regions of lower (larger) chemical loss rates in ion-molecular reactions and leading to the growth (decrease) in electron density (Rishbeth and Garriott, 1969).

As the atomic oxygen is the primary source of ionization at $F$-region heights, and the molecular nitrogen is the primary source of recombination, the change of the $n(\mathrm{O}) / n\left(\mathrm{~N}_{2}\right)$ ratio controls the electron density. The growth (reduction) of this ratio leads to the positive (negative) disturbances in electron density at heights of the ionospheric $F$-region (Rishbeth and Garriott, 1969).

Recent modeling results have shown that the additional eastward electric field such as prompt penetration on its own is unlikely to cause positive ionospheric storms (e.g., Balan et al., 2009). According to the mechanism suggested by Balan et al. (2010), an equatorward neutral wind is required to produce positive ionospheric storms. The mechanical effects of the wind are: (1) to reduce (or stop) the downward diffusion of plasma along the geomagnetic field lines; (2) to raise the ionosphere to higher altitudes with reduced chem- ical loss; and hence accumulate the plasma at altitudes near and above the ionospheric peak centered at around $\pm 30^{\circ}$ magnetic latitudes. Daytime eastward prompt penetration electric fields, if they occur, also shift the Equatorial Ionization Anomaly (EIA) crests to higher than normal latitudes. The difference between the earlier and recent modelling is in latitudinal coverage: the earlier modelling (e.g., Buonsanto, 1999) considered the effects at high- and midlatitudes alone, while Balan et al. (2009) considered lowand mid-latitudes.

\section{Description of the Modeled Event and State- ment of the Problem}

This paper is focused on studies of the ionospheric effects of the September 9-14, 2005 storm sequence. During this period there were several geomagnetic storms following one another: a minor storm on September $9\left(K_{\mathrm{p}}=4+\right)$, a moderate storm on September $10\left(K_{\mathrm{p}}=6-\right)$, and a major storm on September $11\left(K_{\mathrm{p}}=8-\right)$. Figure 1 describes the behavior of the $K_{\mathrm{p}^{-}}, A_{\mathrm{E}^{-}}$, and $D_{\text {st }}$ indices of geomagnetic activity and the index of the solar activity level, $F_{10.7}$ for the period of 8-14 September 2005. It is important to note the high flare activity during the considered period; there were 5 flares on the Sun (on 10 September at 19:10 UT and 21:30 UT, on 11 September at 12:44 UT, on 13 September at 19:19 UT, and on 14 September at 10:05 UT). The considered events occurred at average level of solar activity $\left(F_{10.7} \sim 101-120\right)$.

We use the observations from the ionosondes at Irkutsk and Yakutsk and the ISRs at Millstone Hill and Arecibo. All ionosonde ionogram data have been manually scaled using an interactive ionogram scaling software, SAO Explorer (Khmyrov et al., 2008; Reinisch and Galkin, 2011). In experimental data, we use the diurnal variation on 8 September 2005 as a quiet-time reference (quiet day) for the ISR observations and the monthly median diurnal variation in the case of the ionosonde data.

The majority of numerical modeling studies of the ionosphere's response to geomagnetic storms consider the role of the cross-polar cap potential difference. In addition, models need to include the changes of energy and particle flux of high-energy particle precipitation and the spatial-temporal variations of the Region 2 field-aligned currents (R2 FAC) (Maruyama et al., 2005; Klimenko and Klimenko, 2009). The inclusion of such inputs in global numerical models allows a more accurate description of the Joule heating, effects of penetration of magnetospheric convection electric field to lower latitudes, the overshielding effects, and the effects of the disturbance dynamo electric field. The GSM TIP model (Namgaladze et al., 1988, 1991) with a modified block for the calculation of the electric fields of dynamo and magnetospheric origin (Klimenko et al., 2006, 2007) allows modeling studies with all the drivers described above. The comparison of GSM TIP model calculations for different ionospheric parameters with the observational data at different mid-latitude locations for the period of 8-14 September 2005, presented in earlier studies by Klimenko et al. (2011), has revealed the qualitative agreement. The current investigation focuses on the GSM TIP and IRI model comparison at different longitudes dur- 

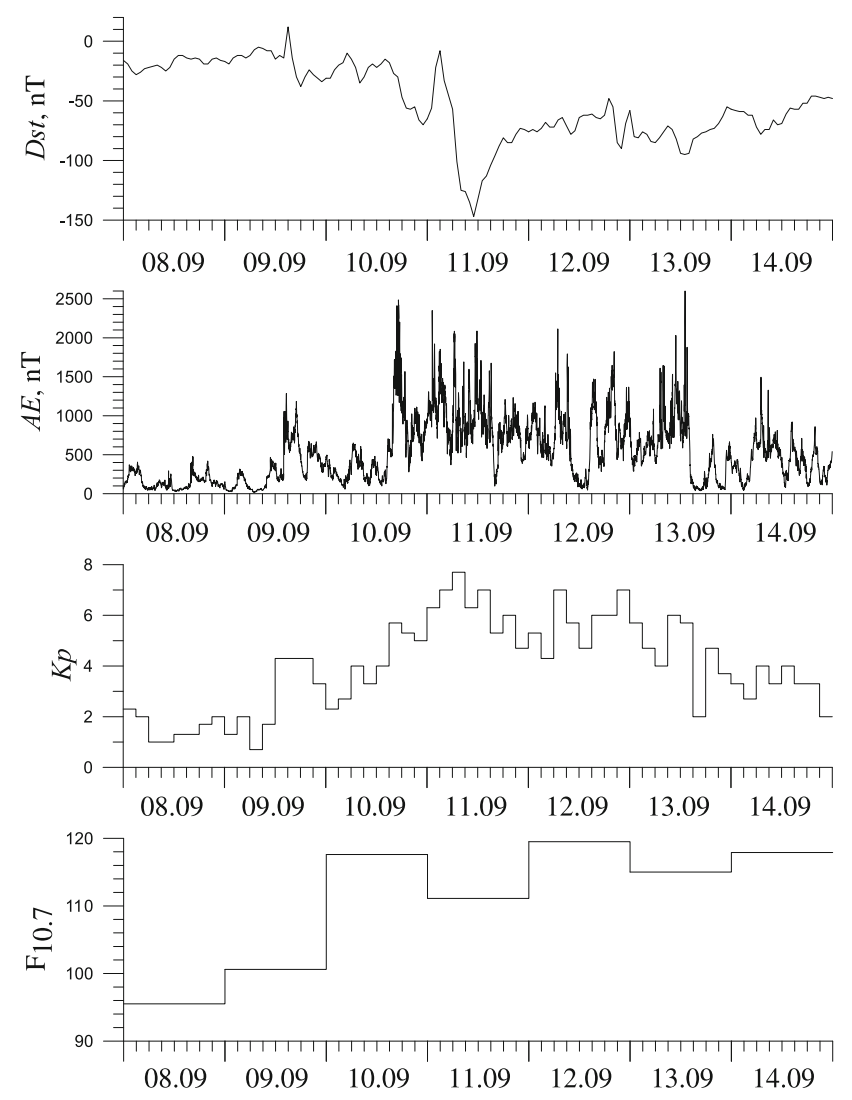

Fig. 1. The behavior of $K_{\mathrm{p}^{-}}, A_{\mathrm{E}^{-}}$and $D_{\mathrm{st}}$-index of geomagnetic activity and the index of solar activity $F_{10.7}$ on September, 8-14, 2005.

ing these geomagnetic storms.

The model run presented here is the same as the one described in detail by Klimenko et al. (2011). The GSM TIP simulation uses as initial condition the values of ionospheric parameters at 06:00 UT on September 9, 2005, obtained for quiet geomagnetic conditions $\left(K_{\mathrm{p}}=0.7\right)$. To calculate the quiet-time behavior of ionospheric parameters, we varied only the $F_{10.7}$ index, while the value of the $K_{\mathrm{p}}$-index remained fixed at the level of 0.7. For quiet conditions, the cross-polar cap potential difference $\Delta \Phi$ was set equal to $35.7 \mathrm{kV}$ at geomagnetic latitudes $\pm 75^{\circ}$, and R2 FAC, $j_{2}$, were set equal to $3 \times 10^{-8} \mathrm{~A} / \mathrm{m}^{2}$ at geomagnetic latitudes $\pm 70^{\circ}$. We are setting the maximum and minimum of electric potential rather than potential difference in the latitudinal circles of $\pm 75^{\circ}$. The distribution of electric potential is defined in such manner that the electric field in the polar cap was directed from dawn to dusk. To be precise, on the dawn side it was set to $17.85 \mathrm{kV}$, and at the dusk side to $-17.85 \mathrm{kV}$. The electric potential at the latitudinal circle $\pm 75^{\circ}$ varies like a sine function. The zeros of this sine function occur on the day and night sides.

In the GSM TIP storm time calculations, several input parameters such as cross-polar cap potential difference, R2 FAC, and auroral particle precipitations varied as a function of the $K_{\mathrm{p}}$-index. The cross-polar cap potential difference was set according to the relation $\Delta \Phi=26.4+13.3 \times K_{\mathrm{p}}$ $(\mathrm{kV})$ (Feshchenko and Maltsev, 2003) at geomagnetic latitudes $\pm 75^{\circ}$. Using the morphological results of Iijima and Potemra (1976) and Kikuchi et al. (2008) we have con- structed the empirical dependences of R2 FAC amplitudes from the $K_{\mathrm{p}}$-index during geomagnetic storms: $j_{2}=2.78 \times$ $10^{-8}+0.32 \times 10^{-8} \times K_{\mathrm{p}}\left(\mathrm{A} / \mathrm{m}^{2}\right)$. We also have included the 30 min time delay of R2 FAC variations with respect to the variations of the cross-polar cap potential difference during the storm (Kikuchi et al., 2008). The flux of precipitating auroral electrons is increased and their spectrum becomes harder with growth of geomagnetic activity. The ratio of the precipitating particles fluxes under the storm and quiet time conditions varied as Flux Storm $/$ Flux $_{\text {Quiet }}=0.55+0.64 \times K_{\mathrm{p}}$.

The GSM TIP model also accounts for the changes in the position of the R2 FAC and high-energy particle precipitation during disturbed conditions. We varied the geomagnetic latitudes of the R2 FAC maximum depending on the changes of a cross-polar cap potential difference: 1) $\pm 70^{\circ}$ at $\left.K_{\mathrm{p}} \leq 3 ; 2\right) \pm 65^{\circ}$ at $\left.3<K_{\mathrm{p}} \leq 6 ; 3\right) \pm 60^{\circ}$ at $6<K_{\mathrm{p}}$ according to the conclusions of Sojka et al. (1994). We also introduced the shift of the storm-time precipitation maximum from the local midnight sector into the local morning sector, and the 30 min delay between the particle precipitation and the changes of cross-polar cap potential difference.

The changes of the polar cap sizes and positions were not taken into account in the GSM TIP model since their inclusion requires development of an absolutely new model. This is related to the tilted dipole approximation of the Earth's magnetic field in the GSM TIP model. Geomagnetic field lines in the polar caps are assumed open, while other geomagnetic field lines are closed. The integration of modeling equations for the thermal plasma of the $F$-region ionosphere and plasmasphere in the GSM TIP model is performed along geomagnetic field lines. For the development of the GSM TIP model the grid of the geomagnetic field lines has been kept fixed. Displacement of the equatorial boundary of the polar cap to the equator should automatically lead to the expansion of the open geomagnetic field lines. The modeling equations solution on open field lines must be consistent with the boundary conditions, describing the regime of the continual escape of thermal plasma (polar wind). The model does not provide the replacement of closed field lines by the open field lines due to the chosen fixed grid. Errors associated with the use of fixed polar cap sizes exist and are not easy to quantify. However, we expect these errors to be not substantial.

\section{Modeled Results and Observations}

The effects on September 9 and 14 are smaller than in other days, therefore, we restricted data presentation to ionospheric effects during the period from 10 to 14 September 2005. Figure 2 shows the global disturbances in the ionospheric $F_{2}$-layer critical frequency, $f_{\mathrm{o}} F_{2}$, obtained by IRI-2000 (left panels) and GSM TIP (right panels) models during this storm sequence at 18:00 UT. We selected the 18:00 UT for illustrative purposes, as the global effects in the ionosphere are most pronounced at that time. Similar but weaker features are obtained as well for other times. The common feature of both model patterns is the presence of the negative disturbances in the northern and southern polar caps and the positive disturbances at equator. On both sides of the geomagnetic equator, the two models show a band of positive disturbances. The latitudinal extent of 
$\Delta$ foF2 (MHz) $\quad 18: 00$ UT $\quad 10.09 .2005$

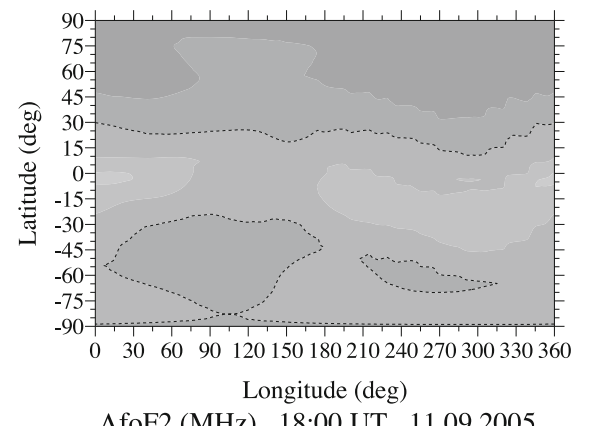

$\Delta$ foF2 (MHz) $\quad$ 18:00 UT 11.09 .2005

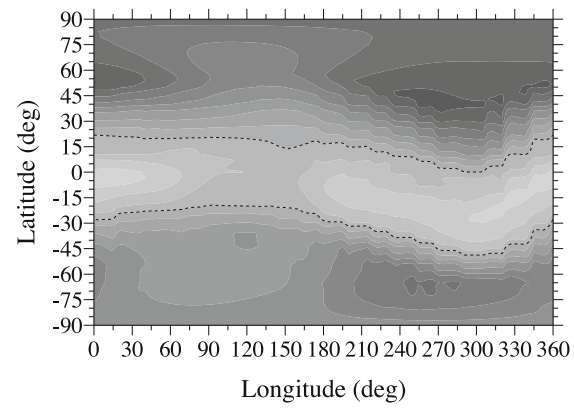

$\Delta$ foF2 (MHz) $18: 00$ UT 12.09 .2005

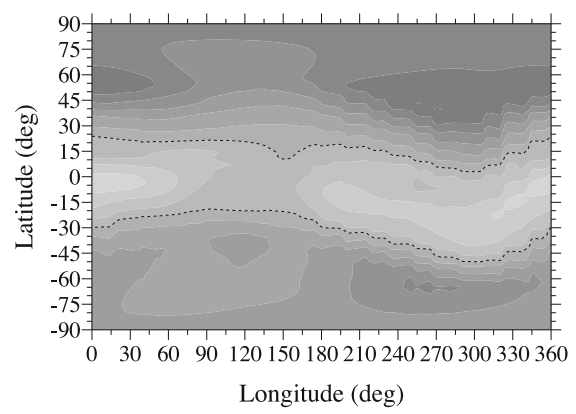

$\Delta \mathrm{foF} 2(\mathrm{MHz}) \quad$ 18:00 UT 13.09 .2005

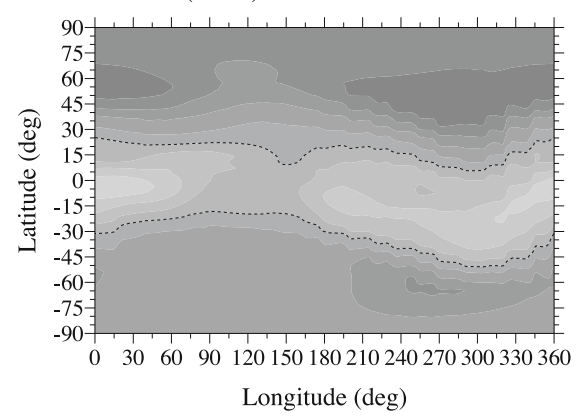

$\Delta$ foF2 (MHz) $\quad$ 18:00 UT $\quad 10.09 .2005$
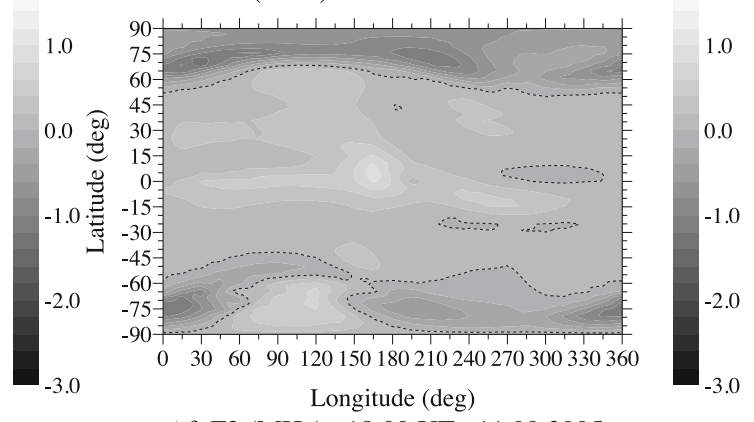

$\Delta$ foF2 (MHz) $18: 00$ UT 11.09 .2005

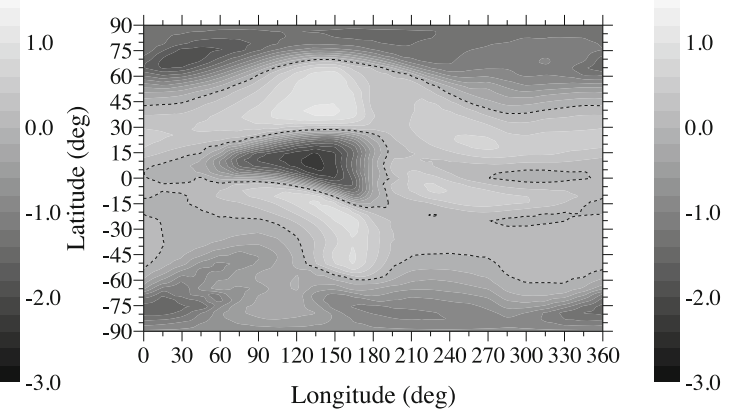

$\Delta$ foF2 (MHz) $18: 00$ UT 12.09 .2005

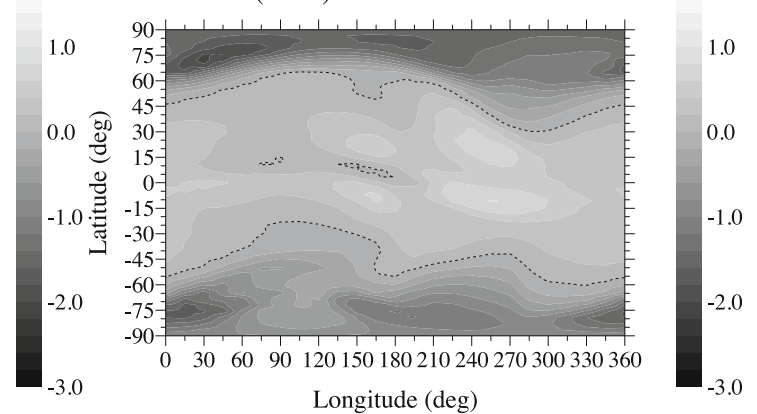

$\Delta$ foF2 (MHz) $\quad$ 18:00 UT 13.09 .2005
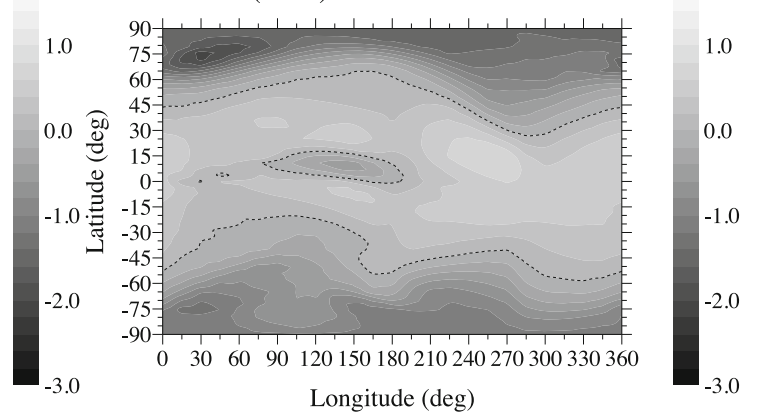

Fig. 2. The global $f_{\mathrm{o}} F_{2}$ disturbances obtained in IRI-2000 model (left panel) and in the model GSM TIP (right panel) during the geomagnetic storm sequence on 10, 11, 12 and 13 September, 2005 at 18:00 UT (from top to bottom).

these positive disturbances in both northern and southern hemispheres is $\sim 15-25^{\circ}$ wider in GSM TIP than in the IRI2000 model. The GSM TIP model also simulates a complex longitudinal structure in positive disturbances, while the IRI-2000 predicted response is mostly symmetric with regards to geomagnetic latitude. The GSM TIP simulation results also show a stronger dependence of positive disturbances on the magnitude of geomagnetic storm, with maximum positive disturbances in $f_{\mathrm{o}} F_{2}$ expected on September 11,2005. The area of negative disturbances seen in the GSM TIP at equatorial and low latitudes is absent in the IRI-2000 band, but coincides with the area of the reduction of the IRI-2000 predicted positive disturbances at the geo-

\section{magnetic equator.}

Figure 3 shows the GSM TIP calculations and IRI-2000 prediction of $f_{\mathrm{o}} F_{2}$ for quiet and storm conditions over different mid-latitude stations. The three upper rows show the $f_{\mathrm{o}} F_{2}$ variations above the stations of the Eastern-Siberian longitudinal chain Norilsk (69.4N, 88.1E), Yakutsk (62.0N, 129.4E) and Irkutsk (52.2N, 104.2E). For September 10, the IRI-2000 model predicts negative $f_{\mathrm{o}} F_{2}$ disturbances above Yakutsk and Irkutsk stations, whereas GSM TIP indicates the negative $f_{\mathrm{o}} F_{2}$ disturbances in the afternoon and the positive disturbances during the night. According to both models, negative $f_{\mathrm{o}} F_{2}$ disturbances are formed above Norilsk. 

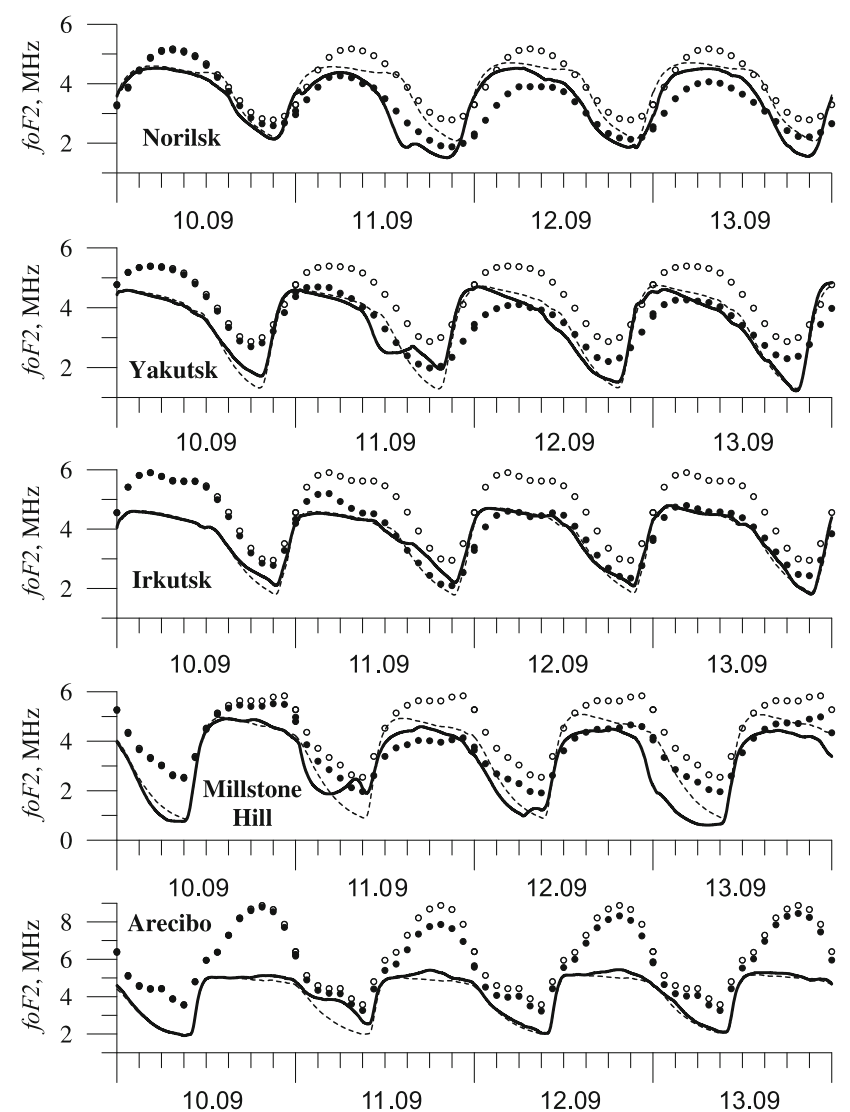

Fig. 3. The calculation results of $f_{\mathrm{o}} F_{2}$ above stations Norilsk, Yakutsk, Irkutsk, Millstone Hill and Arecibo. Dotted and thick lines-GSM TIP model results, light and dark circles-IRI-2000 model results for quiet and storm conditions, respectively.

The two bottom rows in Fig. 3 show the $f_{0} F_{2}$ variations above the stations of the North-American longitudinal chain (Millstone Hill (42.6N, 71.5W) and Arecibo (18.3N, $66.8 \mathrm{~W})$ ). According to both IRI-2000 and GSM TIP, the negative disturbances in $f_{\mathrm{o}} F_{2}$ at Millstone Hill are formed throughout the entire considered period. Exceptions are the disturbances in $f_{\mathrm{o}} F_{2}$ at the daytime of September 10 and the nighttime of September 11 and 12, when GSM TIP shows positive disturbances. IRI-2000 predicts negative $f_{\mathrm{o}} F_{2}$ disturbances above Arecibo for all the days, whereas GSM TIP anticipates positive daytime disturbances for all the days and the positive nighttime disturbances on September 1113. Thus, the IRI-2000 model does not predict any $f_{\mathrm{o}} F_{2}$ storm-time positive disturbances for all the considered stations. Note that the negative disturbances in GSM TIP are a lot smaller than in IRI.

Figures 4 and 5 show the GSM TIP calculations and ionosonde observations of $f_{\mathrm{o}} F_{2}$ and height of $F_{2}$-layer maximum, $h_{\mathrm{m}} F_{2}$ for the quiet and disturbed conditions over Yakutsk and Irkutsk locations, respectively. Since September 10, the GSM TIP disturbances have the same sign with the Yakutsk $f_{\mathrm{o}} F_{2}$ observations, but disagree with the Irkutsk $f_{\mathrm{o}} F_{2}$ behavior, especially with the $f_{\mathrm{o}} F_{2}$ positive daytime disturbance on September 11. Note that the IRI-2000 model does predict $f_{\mathrm{o}} F_{2}$ storm-time negative disturbances only (see Fig. 3). As to the $h_{\mathrm{m}} F_{2}$ behavior, the GSM TIP calculations are in a good agreement with the observations even in

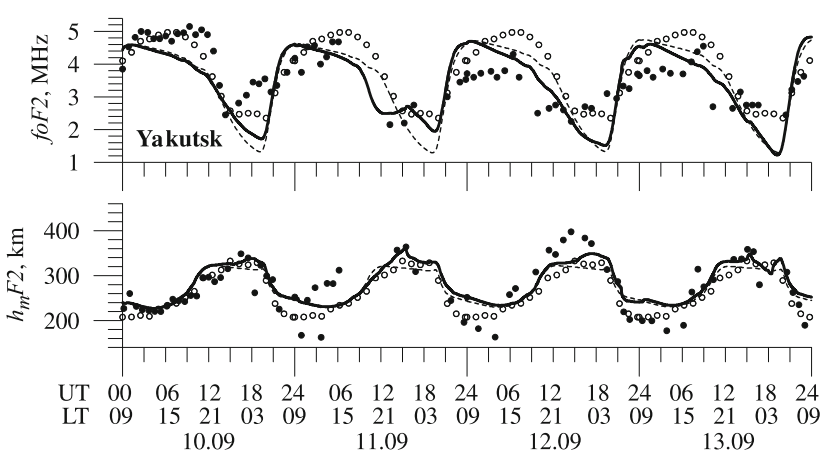

Fig. 4. The behavior of: $f_{\mathrm{o}} F_{2}$ and $h_{\mathrm{m}} F_{2}$ above station Yakutsk. Dotted and thick lines-GSM TIP model results, light and dark circles-digisonde data for quiet and storm conditions, respectively.

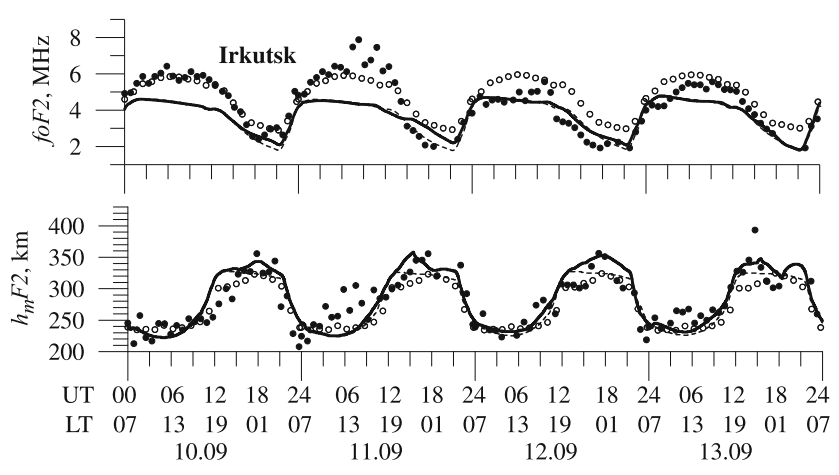

Fig. 5. Same as Fig. 4, but above Irkutsk.

absolute values for both for the quiet and disturbed conditions. The exceptions are: the sharp daytime $h_{\mathrm{m}} F_{2}$ increase by $\sim 100 \mathrm{~km}$ observed at Irkutsk on September 13 , which is not reproduced by GSM TIP; the daytime $h_{\mathrm{m}} F_{2}$ quiet values observed at Yakutsk, which is much smaller than the GSM TIP values. The roles of the different physical mechanisms in the formation of ionospheric disturbances above the Eastern-Siberian stations were considered by Klimenko et al. (2011).

The strong positive disturbance seen in the Irkutsk $f_{\mathrm{o}} F_{2}$ variations on the afternoon of September 11 is absent at first sight at Yakutsk. The reason is that no Yakutsk $f_{\mathrm{o}} F_{2}$ data were available during this period because of strong radio wave absorption, and so it is quite possible that the same positive disturbance existed over Yakutsk. This suggestion is supported in Klimenko et al. (2011) by a similarity in the behavior of the main drivers (variations of electric field, meridional component of thermospheric wind and neutral atmosphere composition) obtained in the GSM TIP calculations for Irkutsk and Yakutsk.

Figures 6 and 8 show the GSM TIP calculated behavior of $f_{\mathrm{o}} F_{2}$ and $h_{\mathrm{m}} F_{2}$, the zonal and meridional components of the electric field, $E_{\text {East }}$ and $E_{\text {North }}$, the meridional component of the thermospheric wind velocity, $U_{n \Theta}$, and the $n(\mathrm{O}) / n\left(\mathrm{~N}_{2}\right)$ ratio at a height of $300 \mathrm{~km}$ for Millstone Hill and Arecibo locations, respectively. The two top plots show the $f_{\mathrm{o}} F_{2}$ and $h_{\mathrm{m}} F_{2}$ variations from Millstone Hill and Arecibo ISRs observation for the quiet and disturbed conditions.

The negative afternoon and evening disturbances in $f_{\mathrm{o}} F_{2}$ are seen in the Millstone Hill digisonde and ISR observa- 


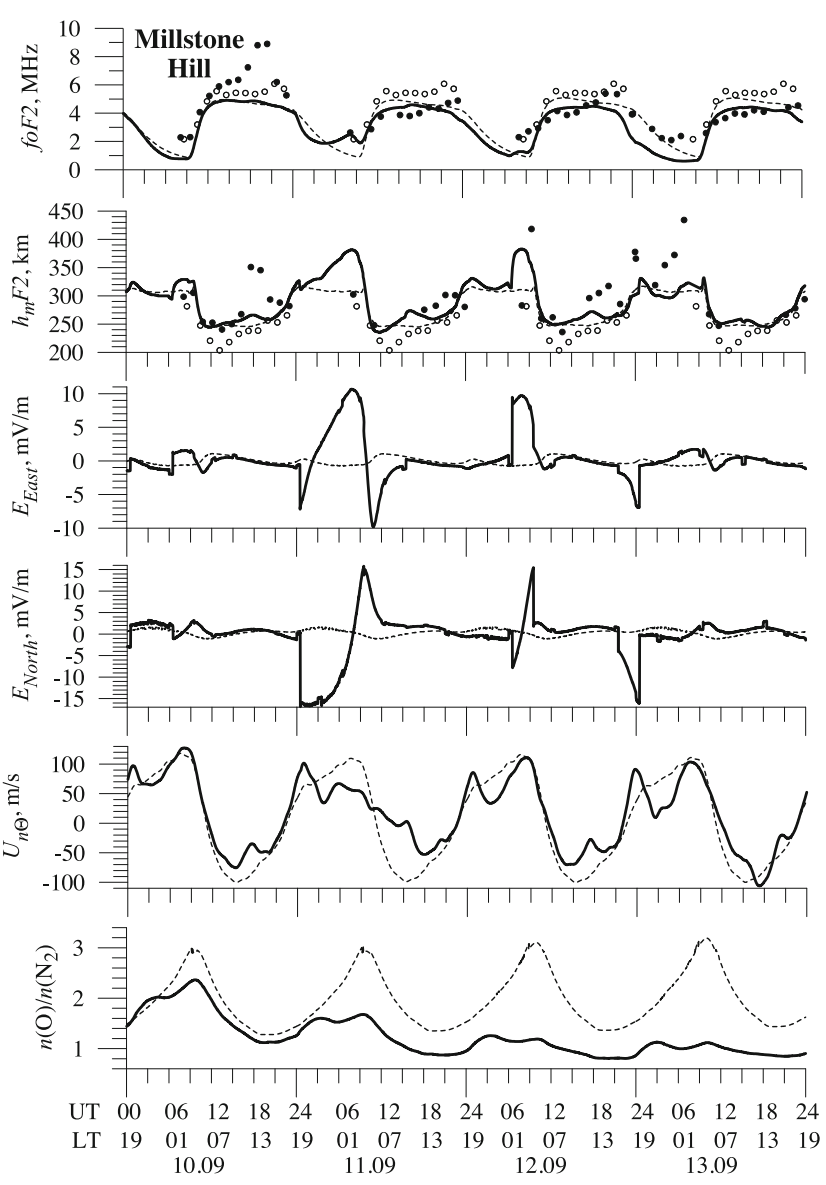

Fig. 6. The behavior of: $f_{\mathrm{o}} F_{2}, h_{\mathrm{m}} F_{2}, E_{\text {East }}, E_{\mathrm{North}}, U_{n \Theta}$ and $n(\mathrm{O}) / n\left(\mathrm{~N}_{2}\right)$ at height of $300 \mathrm{~km}$ above Millstone Hill. Dotted and thick lines-GSM TIP model results, light and dark circles-ISR data for quiet and storm conditions, respectively.

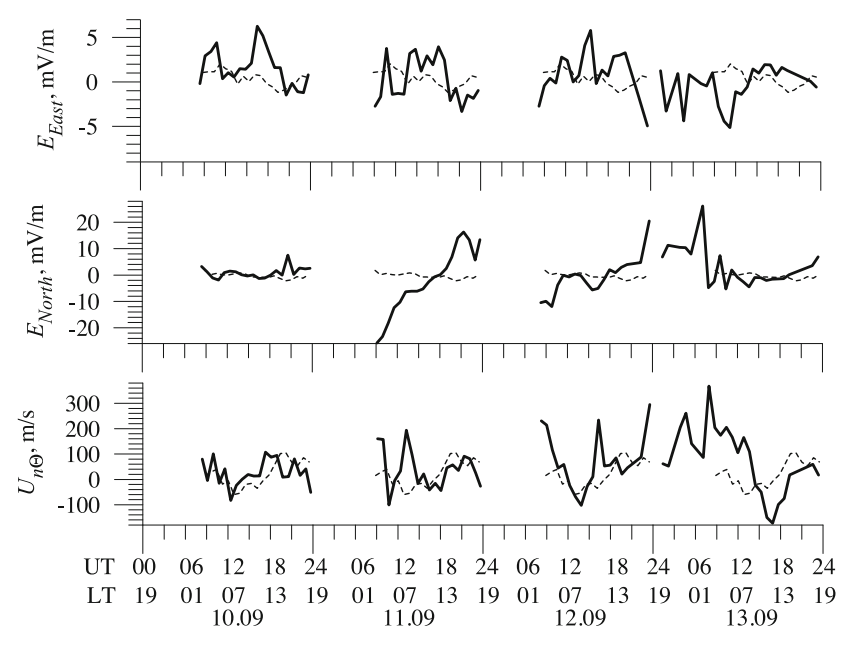

Fig. 7. The behavior of: $E_{\text {East }}, E_{\text {North }}$ and $U_{n \Theta}$ at height of $300 \mathrm{~km}$ above station Millstone Hill. Dotted and solid lines-ISR data for quiet and storm conditions, respectively.

tions during the entire considered period except the positive disturbances in the afternoon on September 10. The sign of these disturbances is in agreement with the GSM TIP calculations; however, the calculated positive and negative disturbances in $f_{\mathrm{o}} F_{2}$ are much weaker than in the observations. IRI-2000 is well reproducing the negative $f_{\mathrm{o}} F_{2}$

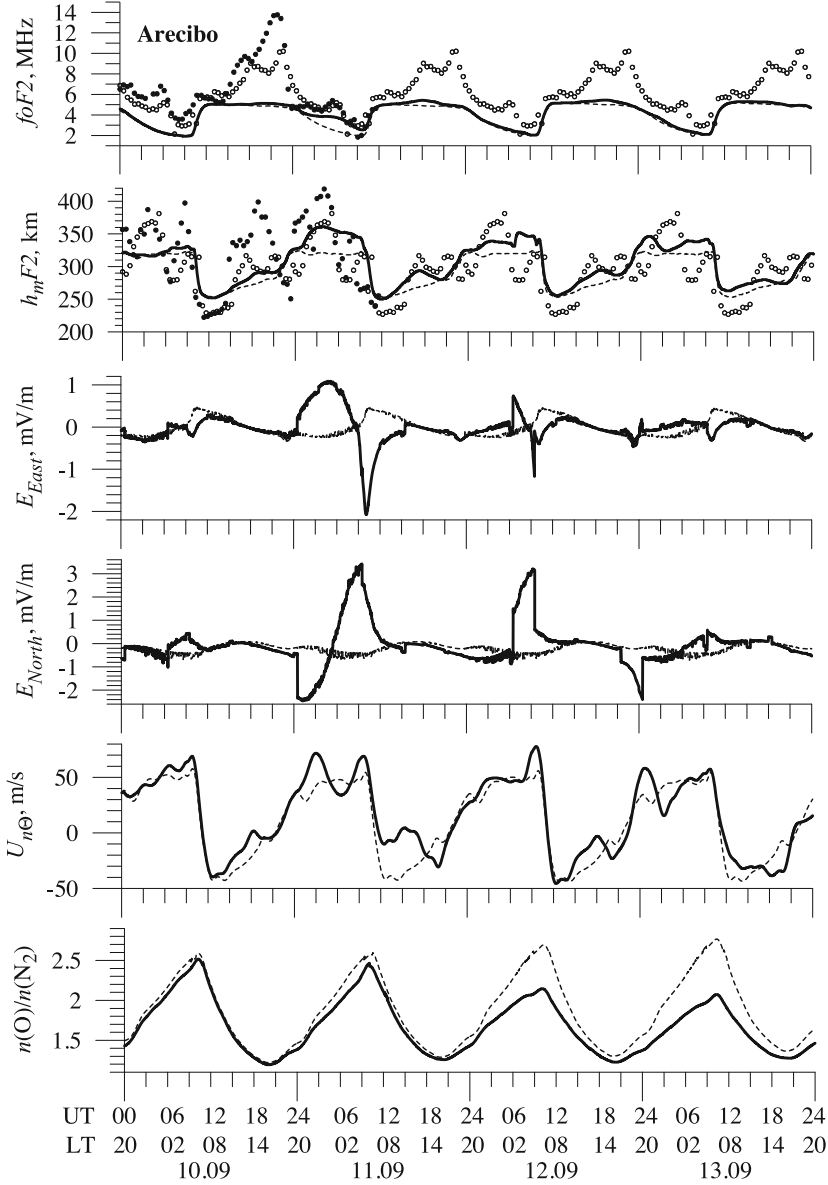

Fig. 8. Same as Fig. 6, but above Arecibo.

disturbances above Millstone Hill (see Fig. 3) but not the positive disturbances.

The positive disturbances are caused by the meridional component of the thermospheric wind. The same conclusion has been made by $\mathrm{Lu}$ et al. (2008) and Klimenko et al. (2011) in the modeling study of the positive phase of the September 10, 2005 ionospheric storm. The GSM TIP reduction of the $n(\mathrm{O}) / n\left(\mathrm{~N}_{2}\right)$ ratio above Millstone Hill should lead to the decrease in the electron density. The resulting effect is defined by the combined action of the thermospheric wind and the neutral atmosphere composition. According to GSM TIP, the contribution of both components of the electric field to this positive disturbance is insignificant. At the same time, the Millstone Hill ISR observations (Fig. 7) show the additional eastward and northward components of electric field, which in the given situation should lead to the positive effect in $f_{\mathrm{o}} F_{2}$. The GSM TIP calculated positive disturbances in $f_{\mathrm{o}} F_{2}$ on September 11 and 12 from 06:00 to 09:00 UT are associated with the action of the additional eastward electric field, whereas the negative disturbances in $f_{\mathrm{o}} F_{2}$ during September 11-13 are associated with the strong reduction of the $n(\mathrm{O}) / n\left(\mathrm{~N}_{2}\right)$ ratio.

According to both the GSM TIP calculations and the observations, the positive disturbances in $h_{\mathrm{m}} F_{2}$ have three peaks: pre-sunrise, daytime, and evening. Both GSM TIP and the observations show that the pre-sunrise peak is as- 
sociated with the additional eastward electric field. The additional equatorward wind amplifies the electric field effect according to the observations. In GSM TIP the day time peak is associated with the increase of the equatorward wind, whereas the observations show the joint contribution of the additional eastward electric field and the equatorward wind. In GSM TIP the evening peak in $h_{\mathrm{m}} F_{2}$ is associated with the effect of the additional equatorward wind which is reduced by the westward electric field.

Only the positive disturbances in $f_{\mathrm{o}} F_{2}$ and $h_{\mathrm{m}} F_{2}$ on September 10 were observed above Arecibo. GSM TIP also shows only the positive disturbances, but the IRI model predicts only the negative disturbances. The afternoon positive disturbances in $f_{\mathrm{o}} F_{2}$ are associated with the additional equatorward thermospheric wind, and the nighttime disturbances are due to the joint action of the equatorward thermospheric wind and both components of the electric field. The reduction of the $n(\mathrm{O}) / n\left(\mathrm{~N}_{2}\right)$ ratio decreases these positive disturbances. According to GSM TIP the positive disturbances in $h_{\mathrm{m}} F_{2}$ have three peaks: pre-sunrise, daytime, and nighttime. The pre-sunrise and nighttime peaks in $h_{\mathrm{m}} F_{2}$ are associated with the joint action of the additional eastward electric field and the additional equatorward thermospheric wind. The day time peak is associated with increase of the equatorward thermospheric wind.

\section{Discussion}

We have compared the GSM TIP calculations, the IRI2000 predictions, and the observations at multiple locations. Figures 3-7 demonstrate that the IRI-2000 predicts the negative disturbances in $f_{\mathrm{o}} F_{2}$ in good agreement with the observations. The exceptions are the observed positive disturbances in $f_{\mathrm{o}} F_{2}$ at Yakutsk, Irkutsk, Millstone Hill and Arecibo. Thus, the main discrepancy between IRI-2000 and the observations is the absence of positive $f_{\mathrm{o}} F_{2}$ disturbances during the considered geomagnetic storms.

Miró Amarante et al. (2007) and Buresova et al. (2010) show that the empirical storm-time ionospheric correction model captures more effectively the negative phases, while electron density enhancement during storms and the transition between the different storm phases is reproduced with less accuracy. This model is not able to reproduce correctly the storm-induced rapid changes in the daily course of $f_{\mathrm{o}} F_{2}$, i.e., the initial rapid positive ionospheric response to the storm onset (Buresova et al., 2010). This is probably due to the insufficient number of observational data used in the development of the empirical storm-time ionospheric correction model. It is likely that the update of this model with increased volume of observational data would improve its performance.

The comparison of the GSM TIP calculations and the observations has revealed both the qualitative agreement and the quantitative disagreement. As suggested in Klimenko et al. (2011), the possible reasons of the differences between the GSM TIP calculations and the observations are the following: the coarse temporal resolution of the model input parameters (e.g., the three-hour $K_{\mathrm{p}}$-index), the use of the dipole approach of geomagnetic field in the GSM TIP model, and the absence of solar flare effects in the model.

The prompt penetration electric fields from high latitudes to the equator occurs due to the failure of shielding conditions of magnetospheric convection electric field by R2 FAC. Vasylíunas (1970) has predicted a $~ 30$ min delay of the shielding effect with respect to the development of the magnetospheric convection electric field. This delay leads to the penetration to the low latitudes of magnetospheric convection electric field at the increasing geomagnetic activity (Kikuchi et al., 2010), and Alfven layer electric field (overshielding effects) at the decreasing geomagnetic activity (Kikuchi et al., 2010). The dependence of the model input parameters on the 3 -hour $K_{\mathrm{p}}$-index allows correct description of the prompt penetration electric field or overshielding only in the first $30 \mathrm{~min}$ after each change in $K_{\mathrm{p}^{-}}$ index.

The use of the dipole approach in the GSM TIP model does not allow consideration of the distortions of the Earth's magnetic field during geomagnetic storms. Tsyganenko et al. (2003) pointed out that in any particle simulation of the inner magnetospheric dynamics during major storms, the use a dipolar or quasi-dipolar magnetic field model is inadequate even at $L \sim 3-4$. The magnetic field should be obtained either using a more realistic empirical model or by means of a fully self-consistent code, based on global particle distributions and externally driven boundary conditions. This approach was used by Li et al. (2011) in a study of the superdense plasma sheet formation during storm-time. At the present stage of GSM TIP model development the use of a realistic geomagnetic field represents a difficult problem which requires the development of an absolutely new model.

Despite the marked imperfections in the present problem statement, we have obtained a qualitative agreement (the disturbances with the same sign) with the observations and confirmed the conclusions about the main formation mechanisms of ionospheric disturbances during the September 10, 2005 storm based on model calculations of Lu et al. (2008). The GSM TIP calculations of the ionospheric effects of the geomagnetic storm sequence show that the positive ionospheric disturbances in $f_{\mathrm{o}} F_{2}$ are mainly caused by the equatorward thermospheric wind and the eastward component of the electric field. This positive effect is reduced by the neutral atmosphere composition change, which is the main mechanism of the negative disturbance formation. The other mechanism is the westward electric field and the poleward thermospheric wind.

\section{Conclusion}

We have presented the modeling of the ionospheric effects of September 9-14, 2005 geomagnetic storm and the comparison of the GSM TIP results and IRI-2000 predictions with the ionosonde and incoherent scatter radar observations at Yakutsk, Irkutsk, Millstone Hill, and Arecibo. The main conclusions are the following.

1. The IRI-2000 model does not reproduce the positive storm time disturbances observed in the mid-latitude $F$-region electron density. The latitudinal extent of the IRI-2000 predicted positive phase is much narrower than in GSM TIP, while the magnitude of the response is smaller than in GSM TIP. The GSM TIP simulation, 
in turn, strongly underestimates the observed positive phase of the ionospheric storm.

2. The GSM TIP disturbances are of the same sign as the ionosonde and ISR observations. The differences between storm and quiet time are always smaller in the GSM TIP model than in the observational data. The largest discrepancies occurred at Irkutsk during daytime on September 11, 2005. We suggest that the causes of the differences between model calculations and data were the coarse (3-hour) resolution of the model drivers, the dipole approximation of geomagnetic field in GSM TIP, and also the possible effects of solar flares which are not included in GSM TIP calculations.

3. The analysis of the observations and GSM TIP calculations has shown that the positive ionospheric storm is mainly caused by the equatorward meridional thermospheric wind, and can be further enhanced by the eastward component of electric field. This positive effect is reduced by the change of the neutral atmosphere composition which is the main formation mechanism of the negative disturbances in $f_{\mathrm{o}} F_{2}$.

Acknowledgments. The authors acknowledge the Irkutsk and Yakutsk digisonde teams and Arecibo and Millstone Hill ISR teams for processing the data and making the experimental data available. We express our gratitude to Prof. Bodo Reinisch for his help in evaluating this paper. These investigations were carried out with the financial support of the Russian Foundation for Basic Research (RFBR)—Grant No. 08-05-00274.

\section{References}

Balan, N., K. Shiokawa, Y. Otsuka, S. Watanabe, and G. J. Bailey, Super plasma fountain and equatorial ionization anomaly during penetration electric field, J. Geophys. Res., 114, A03310, doi:10.1029/2008JA013768, 2009.

Balan, N., K. Shiokawa, Y. Otsuka, T. Kikuchi, D. Vijaya Lekshmi, S. Kawamura, M. Yamamoto, and G. J. Bailey, A physical mechanism of positive ionospheric storms at low latitudes and midlatitudes, J. Geophys. Res., 115, A02304, doi:10.1029/2009JA014515, 2010.

Bilitza, D., International Reference Ionosphere 2000, Radio Sci., 36, 261275, 2001.

Bilitza, D., International Reference Ionosphere 2000: Examples of improvements and new features, Adv. Space Res., 31, 757-767, 2003.

Buonsanto, M. J., Ionospheric storms-a review, Space Sci.Rev., 88, 563601, 1999.

Buresova, D., L.-A. McKinnell, T. Sindelarova, and B. A. De La Morena, Evaluation of the STORM model storm-time corrections for middle latitude, Adv. Space Res., 46, 1039-1046, 2010.

Feshchenko, E. Yu. and Yu. P. Maltsev, Relations of the polar cap voltage to the geophysical activity, Proc. XXVI Annual Seminar "Physics of Auroral Phenomena", pp. 59-61, February 25-28, 2003, Apatity, PGI KSC RAS, 2003.

Forbes, J. M., Dynamics of the thermosphere, J. Meteor. Soc. Jpn., 85B, 193-213, 2007.

Iijima, T. and T. A. Potemra, Field-aligned currents in the dayside cusp observed by triad, J. Geophys. Res., 81, 5971-5979, 1976.

Khmyrov, G. M., I. A. Galkin, A. V. Kozlov, B. W. Reinisch, J. McElroy, and C. Dozois, Exploring digisonde ionogram data with SAO-X and DIDBase, Radio Sounding and Plasma Physics, AIP Conf. Proc., 974, 175-185, 2008.

Kikuchi, T., K. K. Hasimoto, and K. Nozaki, Penetration of magnetospheric electric fields to the equator during a geomagnetic storm, $J$. Geophys. Res., 113, doi:10.1029/2007JA012628, 2008.
Kikuchi, T., Y. Ebihara, K. K. Hashimoto, R. Kataoka, T. Hori, S. Watari, and N. Nishitani, Penetration of the convection and overshielding electric fields to the equatorial ionosphere during a quasiperiodic DP 2 geomagnetic fluctuation event, J. Geophys. Res., 115, A05209, doi:10.1029/2008JA013948, 2010.

Klimenko, M. V. and V. V. Klimenko, Numerical simulation effects of magnetospheric convection, particle precipitation and field aligned currents of the second region during sequence of geomagnetic storms September 9-14, 2005, KSTU News, Kaliningrad, KSTU, 16, 220-228, 2009 (in Russian).

Klimenko, M. V., V. V. Klimenko, and V. V. Bryukhanov, Numerical simulation of the electric field and zonal current in the Earth's ionosphere: The dynamo field and equatorial electrojet, Geomagn. Aeron., 46, 457466, 2006.

Klimenko, M. V., V. V. Klimenko, and V. V. Bryukhanov, Numerical modeling of the equatorial electrojet UT-variation on the basis of the model GSM TIP, Adv. Radio Sci., 5, 385-392, 2007.

Klimenko, M. V., V. V. Klimenko, K. G. Ratovsky, and L. P. Goncharenko, Ionospheric effects of geomagnetic storm sequence on September 9-14, 2005, Geomagn. Aeron., 51(3), 2011 (in print).

Klimenko, V. V. and A. A. Namgaladze, Ionospheric effects of meridional electric fields, in Ionosphere Variations During Magnetospheric Disturbances, Moscow, Nauka, 3-10, 1980 (in Russian).

Li, W., J. Raeder, M. F. Thomsen, and B. Lavraud, The formation of superdense plasma sheet in association with the IMF turning from northward to southward, J. Geophys. Res., 116, doi:10.1029, 2011 (in print).

Lu, G., L. P. Goncharenko, A. D. Richmond, R. G. Roble, and N. Aponte, A dayside ionospheric positive storm phase driven by neutral winds, $J$. Geophys. Res., 113, doi:10.1029/2007JA012895, 2008.

Maruyama, N., S. Watanabe, and T. J. Fuller-Rowell, Dynamic and energetic coupling in the equatorial ionosphere and thermosphere, J. Geophys. Res., 108(A11), 1396, doi:10.1029/2002JA009599, 2003.

Maruyama, N., A. D. Richmond, T. J. Fuller-Rowell, M. V. Codrescu, S. Sazykin, F. R. Toffoletto, R. W. Spiro, and G. H. Millward, Interaction between direct penetration and disturbance dynamo electric fields in the storm-time equatorial ionosphere, Geophys. Res. Lett., 32, doi:10.1029/2005GL023 763, 2005.

Mayr, H. G. and H. Volland, Magnetic storm characteristics of the thermosphere, J. Geophys. Res., 78, 2251-2264, 1973.

Miró Amarante, G., M. Cueto Santamaría, K. Alazo, and S. M. Radicella, Validation of the STORM model used in IRI with ionosonde data, $A d v$. Space Res., 39, 681-686, 2007.

Namgaladze, A. A., Yu. N. Korenkov, V. V. Klimenko, I. V. Karpov, F. S. Bessarab, V. A. Surotkin, T. A. Glushenko, and N. M. Naumova, Global model of the thermosphere-ionosphere-protonosphere system, Pure Appl. Geophys., 127, 219-254, 1988.

Namgaladze, A. A., Yu. N. Korenkov, V. V. Klimenko, I. V. Karpov, V. A. Surotkin, and N. M. Naumova, Numerical modelling of the thermosphere-ionosphere-protonosphere system, J. Atmos. Terr. Phys., 53, 1113-1124, 1991.

Reinisch, B. W. and I. A. Galkin, Global Ionospheric Radio Observatory (GIRO), Earth Planets Space, 63(4), 377-381, 2011.

Rishbeth, H. and K. Garriott, Introduction to Ionospheric Physics, Academic Press, New York, 1969.

Schunk, R. W., Response of the ionosphere-thermosphere system to magnetospheric forcing, Adv. Space Res., 10(6), 133-142, 1990.

Sojka, J. J., R. W. Schunk, and W. F. Denig, Ionospheric response to the sustained high geomagnetic activity during the March ' 89 great storm, J. Geophys. Res., 99, 21341-21352, 1994.

Tsyganenko, N. A., H. J. Singer, and J. C. Kasper, Storm-time distortion of the inner magnetosphere: How severe can it get?, J. Geophys. Res., 108(A5), 1209, doi:10.1029/2002JA009808, 2003.

Vasylíünas, V. M., Mathematical models of magnetosphere convection and its coupling to the ionosphere, in Particles and Fields in the Magnetosphere, edited by McCormac, B. M., pp. 60-71, D. Reidel, Dordrecht, 1970.

M. V. Klimenko (e-mail: maksim.klimenko@mail.ru), V. V. Klimenko, K. G. Ratovsky, and L. P. Goncharenko 